\title{
Enhancing Effect of Medium-Chain Triglycerides on Intestinal Absorption of $d-\alpha$-Tocopherol Acetate from Lecithin-Dispersed Preparations in the Rat
}

\author{
Eiji FUKUI, Harumi KUROHARA, Akira KAGEYU, Yuji KUROSAKI, Taiji NAKAYAMA \\ and Toshikiro KIMURA*
}

Faculty of Pharmaceutical Sciences, Okayama University, Tsushima-naka, Okayama, 700, Japan

(Received July 20, 1988)

\begin{abstract}
The effect of formulations of lecithin-dispersed preparation on the absorption of $d$ - $\alpha$-tocopherol acetate (VEA) from the small intestine was investigated in rats. When lecithin-dispersed preparations containing VEA or polysorbate 80 (PS-80)-solubilized solution of VEA were intraduodenally administered, VEA was hydrolyzed to $d$ - $\alpha$-tocopherol (VE) and was not detected in the plasma nor in the thoracic lymph. The maximum plasma concentration $\left(C_{\max }\right)$ of VE after the intraduodenal administration of a preparation consisting of VEA, soybean phosphatidylcholine (PC) and medium-chain triglycerides (MCTG) (VEA/PC/MCTG, 5/16/1 by weight) was highest among the VEA preparations, and PS-80-solubilized solution gave the lowest $C_{\max } . A U C$ of VE up to $24 \mathrm{~h}$ was also increased by the addition of MCTG to VEA/PC preparation.

In the thoracic duct-fistula rat, the transport of $\mathrm{VE}$ into the thoracic lymph was increased by the administration of the VEA/PC/MCTG preparation significantly more than the VEA/PC preparation; the cumulative amounts of VE recovered in the thoracic lymph up to $24 \mathrm{~h}$ were $23.2 \pm 0.5 \%$ and $10.9 \pm 1.5 \%$ of dose, respectively. The plasma concentration of VE was not increased in the thoracic duct-fistula rat even after the intraduodenal administration of VEA preparations, suggesting that VE is not transported directly to the systemic circulation, but by way of the lymphatic route.

The lymphatic transport of VE following the intraduodenal administration of VEA/PC/MCTG preparation was markedly diminished by the simultaneous administration of Pluronic L-81 emulsion, an inhibitor of chylomicron formation. It is suggested that the chylomicron is essential to the lymphatic transport of VE from VEA preparations.
\end{abstract}

Keywords $-d$ - $\alpha$-tocopherol acetate; intestinal absorption; $d$ - $\alpha$-tocopherol; lymphatic transport; lecithin-dispersed preparation; medium-chain triglyceride; rat

\section{Introduction}

$d$ - $\alpha$-Tocopherol (VE) is a potent biological antioxidant and plays an important role in the stabilization of biological membranes. In oral administration for therapy against VE deficiency, $d$ - $\alpha$-tocopherol acetate (VEA) is often used, since the ester is more stable against the oxidation. The absorption mechanism of VEA has been reported as follows.VEA is first hydrolyzed in the intestinal lumen by the presence of bile ${ }^{1)}$ and pancreatic juice. ${ }^{2,3)} \mathrm{VE}$ formed is dispersed by the formation of micelles with bile salts, and then is absorbed from the intestinal tract by a passive diffusion mechanism. ${ }^{4}$ Since waterinsoluble drugs are poorly absorbed from the gastrointestinal tract in general, development of dosage forms ${ }^{5)}$ and use of the absorption promoters $^{6,7)}$ have been extensively studied to im- prove their absorption. The lecithin-dispersed preparations such as liposomes and lipid microspheres, which have attracted special interest because of their adaptation to targeting therapy, are also one of the expected oral dosage forms. Recently, we reported that a lecithin-dispersed aqueous preparation containing medium-chain triglycerides (MCTG) gave a lasting high VE plasma concentration in comparison with polysorbate 80 (PS-80)-solubilized aqueous solution and soybean-oil solution of VEA in rats, ${ }^{8)}$ and the importance of lecithin in enhancing bioavailability of the vitamin is suggested.

The present study deals with the effect of lecithin-dispersed formulations on VEA absorption together with the transport route of VE, the portal venous or the thoracic lymphatic route, following the intraduodenal administration of VEA preparations containing different amounts

* To whom correspondence should be addressed. 
of MCTG in rats.

\section{Materials and Methods}

Materials - VEA, VE, $d l$-tocol (used as an internal standard) and MCTG (fatty acid composition: capric acid/caprylic acid, 1:3) were supplied by Eisai Co., Tokyo. Soybean phosphatidylcholine (PC) was supplied by Nippon Shoji Co., Osaka, and its phosphatidylcholine content was about $95 \%$. Pluronic L-81 (L-81) was supplied by BASF Wyandotte Co., Parsippany, NJ. All other reagents were reagent grade commercial products.

Preparation of Dosage Forms - A chloroform solution containing PC (160 $\mathrm{mg})$ and VEA (50 $\mathrm{mg}$ ), without or with MCTG $(5,10,50$ or $100 \mathrm{mg}$ ) was evaporated under nitrogen gas using a rotary evaporator at room temperature in a round bottomed flask. The thin lipid-film formed on the wall of the flask was hydrated in $10 \mathrm{ml}$ of distilled water and was vortexed for 10 min at room temperature, followed by sonication (Ohtake Sonicator-5202, Tokyo; $20 \mathrm{kHz}$, $100 \mathrm{~W}$ ) for $15 \mathrm{~min}$ on ice. The resultant lecithindispersed preparations were used for all the experiments. A $1.6 \%$ PS-80-solubilized solution was prepared from $50 \mathrm{mg}$ VEA and $10 \mathrm{ml}$ of $1.6 \%$ PS-80 solution by sonication for $30 \mathrm{~min}$ at room temperature.

L-81 emulsion was prepared from a mixed solution of PC (16 mg) and L-81 (150 mg) in chloroform. Chloroform was removed by a rotary evaporator. The thin lipid-film thus formed was resuspended in $3 \mathrm{ml}$ of distilled water and the suspension was sonicated for $3 \mathrm{~min}$ on ice.

Absorption Studies - Male Wistar albino rats weighing $180-250 \mathrm{~g}$ were fasted for $12 \mathrm{~h}$ prior to VEA administration, but had free access to water. Rats were anesthetized with sodium pentobarbital $(45 \mathrm{mg} / \mathrm{kg}$, i.p.) and a blood sample $(300 \mu 1)$ was obtained from the tail artery. Immediately after the collection of 0 -time blood sample, the duodenum was exposed through an incision in the upper half of midline and VEA preparations $(10 \mathrm{mg} / \mathrm{kg})$ were administered intraduodenally followed by closing of the incision. Blood samples were collected at $3,6,9,12,15$, and $24 \mathrm{~h}$ after the administra- tion. After awakening, the rat was left undisturbed. The rectal temperature was maintained at $37^{\circ} \mathrm{C}$ throughout the experiment.

Lymphatic Transport of VE - Under pentobarbital anesthesia, the thoracic lymph duct was cannulated with a clear vinyl tube (i.d., 0.58 mm; o.d., $0.96 \mathrm{~mm}$; Dural Plastics, Australia) for the collection of lymph according to the method of Bollman et al. ${ }^{\text {9) }}$ with a slight modification. A heparin-filled vinyl tube was threaded about $3 \mathrm{~mm}$ into the thoracic lymph duct and a lump of tissue cement, Aron Alpha (Toagosei Chemicals Co., Tokyo), was applied to fix the cannula. After the operation was completed, VEA preparations were intraduodenally administered and then lymph and the blood samples were collected periodically. Volume of these lymph samples was determined by weight measurement.

\section{Effect of L-81 on Lymphatic Transport of} VE - After the thoracic lymph duct was cannulated, VEA/PC/MCTG preparation and 0.4 $\mathrm{ml}$ of L-81 emulsion were intraduodenally administered simultaneously. Then lymph and blood samples were collected periodically.

Assay Procedure for VE and VEA - The blood sample was centrifuged at $3000 \mathrm{rpm}$ for 1 min and $100 \mu 1$ of the plasma was acquired. Then, $100 \mu l$ of water and $300 \mu 1$ of ethanol were added to this plasma. After vortexing well, $n$-hexane containing a known concentration of $d l$-tocol was added to the mixture, followed by shaking and centrifugation. The organic layer was sampled out and was evaporated to dryness. The residue was redissolved in pure $n$-hexane and the solution was used as an analytical sample, which was stored at $-20^{\circ} \mathrm{C}$. The lymph sample was extracted by the same procedure as the plasma sample. Samples from the plasma and the lymph were analyzed by a HPLC (LC-5A, Shimadzu, Kyoto) equipped with a fluorescence HPLC monitor, RF-535 (Shimadzu; the excitation and emission wavelengths were 290 and $325 \mathrm{~nm}$, respectively) which was operated by a normal phase. The analytical column was a Polygosil 60-5 (4 mm i.d. $\times 250 \mathrm{~mm}$, Chemco Scientific Co., Osaka). The mobile phase was composed of $n$-hexane-isopropanol (100: 0.6 by volume), and the flow rate was 1.8 
$\mathrm{ml} / \mathrm{min}$. VE and VEA concentrations were calculated from the ratio of the peak area against that of $d l$ - tocol.

Statistical Analysis - Statistical analysis was carried out by a Student's $t$ - test.

\section{Results}

\section{Plasma Concentration of the Endogenous VE}

In order to estimate the plasma concentration of VE after the administration of VEA preparations, the time course of the plasma concentration of VE was measured for $12 \mathrm{~h}$ in fasted rats. As shown in Fig. 1, endogenous VE in the plasma was almost constant, ranging from 3 to 4 $\mu \mathrm{g} / \mathrm{ml}$. Consequently, it may be quite all right to consider that the plasma concentration of endogenous VE is equal to that before the administration of VEA in each rat. Thus, the increase in the plasma concentration of $\mathrm{VE}$ after the administration of VEA preparations was estimated by subtracting VE concentration just before the administration from each determined value.

\section{Structure of VEA Preparations}

Electron microscopic observation showed that VEA/PC $(5 / 16)$ preparation had the structure of multilamellar liposomes and that the addition of MCTG changed the structure to larger droplets with few or no bilayer structures around them.

The particle size of the VEA/PC/MCTG $(5 / 16 / 1)$ preparation was $1-2 \mu \mathrm{m}$ on measurement with a Coulter counter (Model TA-II,

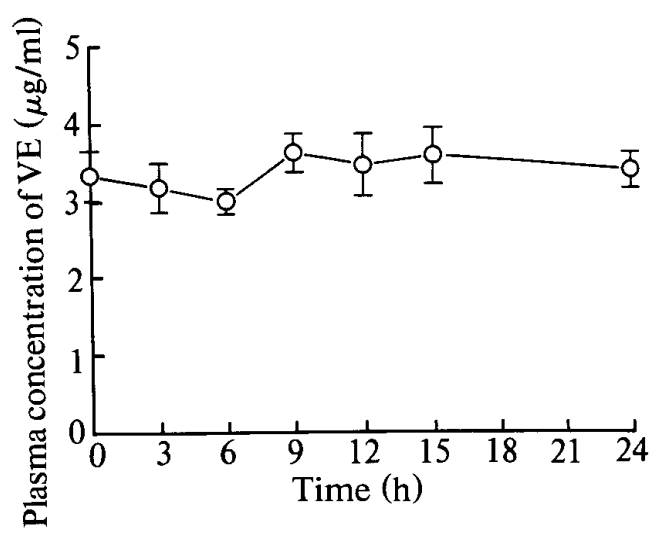

Fig. 1. Plasma Concentration of Endogenous VE in Fasted Rats

Results are expressed as the mean \pm S.E. of 4 rats.

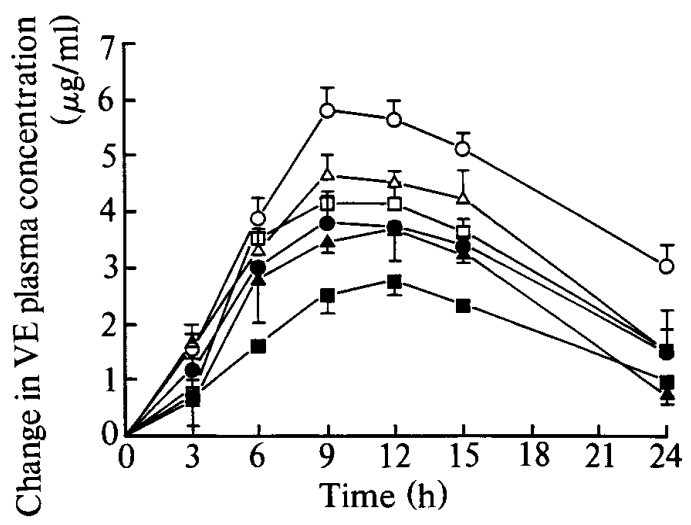

Fig. 2. Changes in Plasma Concentration of VE after Intraduodenal Administration of VEA Preparations in Rats

1.6\% PS-80-solubilized preparation: $\mathrm{VEA} / \mathrm{PC} /$ MCTG preparations $(5 \mathrm{mg} / 16 \mathrm{mg} / x \mathrm{mg}$ in $1 \mathrm{ml}): \bullet, x=$ $0 ; \Delta, x=0.5 ; \bigcirc, x=1 ; \Delta, x=5 ; \square, x=10$. Dose of VEA was $10 \mathrm{mg} / \mathrm{kg}$. Results are expressed as the mean \pm S.E. of $3-5$ rats.

Coulter Electronics Inc., Fla.), but it changed to about $80 \mathrm{~nm}$ in $5 \mathrm{mM}$ sodium taurocholate solution on measurement with a dynamic lightscattering instrument (Model DLS-700, Otsuka Electronics Co., Hirakata). The turbidity of VEA/PC/MCTG $(5 / 16 / 10)$ preparation measured at $600 \mathrm{~nm}$ was much higher than that of the $(5 / 16 / 1)$ preparation, even if $5 \mathrm{~mm}$ sodium taurocholate was present. Thus, it was indicated that the particle size of the former preparation was larger than the latter.

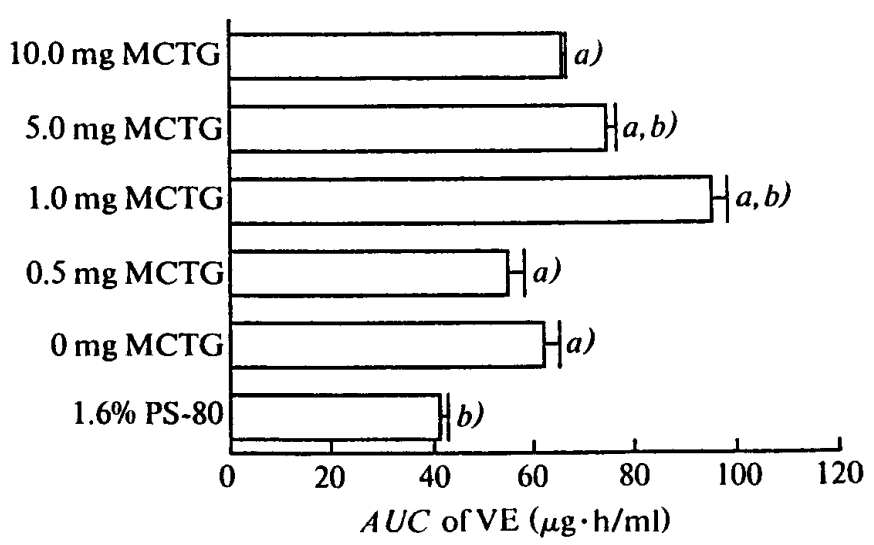

Fig. 3. $A U C$ of Curves Shown in Fig. 2 up to $24 \mathrm{~h}$

Columns were assigned by the amount of MCTG in 1 $\mathrm{ml}$ of the preparation. Results are expressed as the mean + S.E. of $3-5$ rats. a) Significantly different from $1.6 \%$ PS-80, $p<0.05$. b) Significantly different from $0 \mathrm{mg}$ MCTG, $p<0.01$. 
Time Course of Plasma Concentration of VE Following Intraduodenal Administration of VEA Preparations

The changes in VE plasma concentration and their $A U C$ from 0 to $24 \mathrm{~h}$ after the intraduodenal administration of VEA preparations are shown in Figs. 2 and 3, respectively. $C_{\max }$ for VEA/PC/MCTG $(5 / 16 / 1)$ preparation $(5.7 \pm 0.2 \mu \mathrm{g} / \mathrm{ml})$ was highest in the VEA preparations, whereas PS-80-solubilized solution gave the lowest $C_{\max }$. When VEA preparations were estimated by $A U C$ up to 24 h, VEA preparations containing MCTG $(5 / 16 / 1$ or $5 / 16 / 5)$ were significantly better than the VEA/PC preparation. Moreover, VE plasma concentrations for lecithin-dispersed VEA preparations were not decreased to the level of the endogenous one even after $24 \mathrm{~h}$.

Plasma Concentration of VE Following Intraduodenal Administration of VEA Preparations in Thoracic Duct-Fistula Rats

In the thoracic lymph-fistula rat, no significant change in the plasma concentration of $\mathrm{VE}$ after the intraduodenal administration of VEA preparations was recognized (Fig. 4), suggesting that VE was not directly transported into the portal vein after the small-intestinal absorption.

Lymphatic Transport of VE Following Intraduodenal Administration of VEA Preparations

The rate of VE appearance in the thoracic

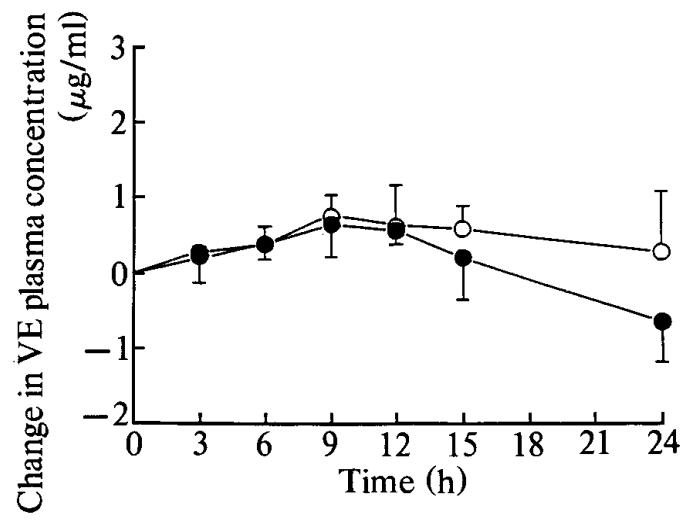

Fig. 4. Changes in Plasma Concentration of VE after Intraduodenal Administration of VEA as VEA/PC/MCTG $(5 / 16 / 1)(\bigcirc)$ and VEA/PC $(5 / 16)(\bullet)$ Preparations in Thoracic Duct-Fistula Rats

Dose of VEA was $10 \mathrm{mg} / \mathrm{kg}$. Results are expressed as the mean \pm S.E. of 4 rats. lymph after the intraduodenal administration of VEA preparations is shown in Fig. 5. The transport rate of $\mathrm{VE}$ was not significantly different in either VEA preparation up to $6 \mathrm{~h}$, after which it reduced in the case of $\mathrm{VEA} / \mathrm{PC}$ preparation. However, in VEA/PC/MCTG preparation, the maximum transport rate of VE was observed between 6 and $9 \mathrm{~h}$. The maximum transport rates of $V E$ were $69.3 \pm 7.4$ and $33.0 \pm 6.0 \mu \mathrm{g} / \mathrm{h}$ for VEA/PC/MCTG and VEA/PC preparations, respectively. The cumulative amounts of $\mathrm{VE}$ recovered in the thoracic lymph up to $24 \mathrm{~h}$ were significantly different: $23.2 \pm 0.5 \%$ and $10.9 \pm 1.5 \%$ of dose for $\mathrm{VEA} / \mathrm{PC} / \mathrm{MCTG}$ and VEA/PC preparations, respectively.

\section{Effect of L-81 on Lymphatic Transport of VE}

It has been reported that L-81, a lipophilic non-ionic surfactant, inhibited the synthesis and the transport of chylomicrons in the smallintestinal mucosa during lipid absorption, ${ }^{10)}$ resulting in the inhibition of lymphatic transport of triglycerides ${ }^{11)}$ and cholesterol. ${ }^{12)}$ To investigate the absorption mechanism of VE from VEA/PC/MCTG preparation, the effect of simultaneous administration of L-81 emulsion on the lymphatic transport rate and/or the plasma concentration of VE was examined in the thoracic lymph-fistula rat. The results are shown in Fig. 6 (A) and (B), respectively. In a control experiment, the liposome of $\mathrm{PC}$ alone, instead of

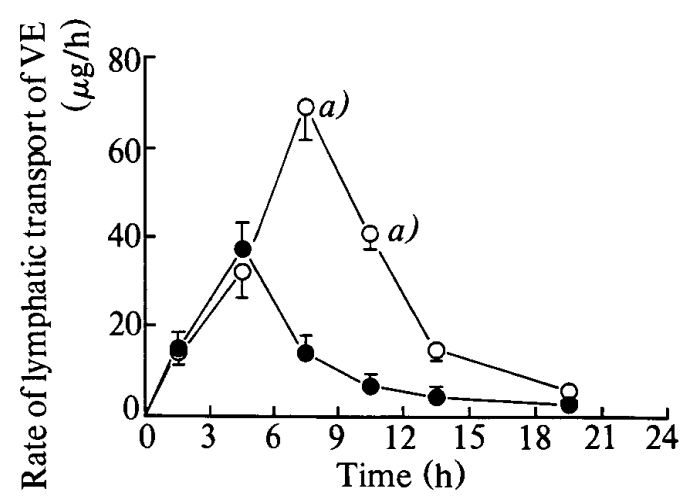

Fig. 5. Transport Rate in Thoracic Lymph of VE after Intraduodenal Administration of VEA as VEA/PC/MCTG $(5 / 16 / 1)(\bigcirc)$ and VEA/PC (5/16) (๑) Preparations in Thoracic Duct-Fistula Rats

Dose of VEA was $10 \mathrm{mg} / \mathrm{kg}$. Results are expressed as the mean \pm S.E. of 4 rats. Statistically-significant difference at each time is shown as $a)(p<0.001)$. 

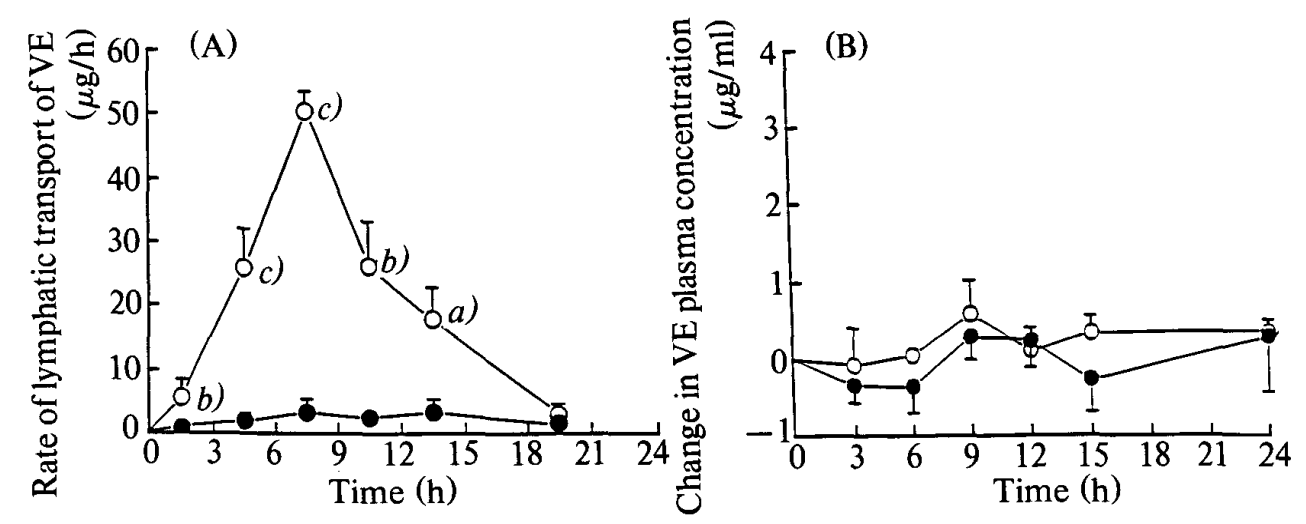

Fig. 6. Transport Rate in Thoracic Lymph (A) and Change in Plasma Concentration (B) of VE after Intraduodenal Administration of VEA as VEA/PC/MCTG (5/16/1) Preparation with $(\bullet, n=7)$ or without $(O, n=4)$ L-81 in Thoracic DuctFistula Rats

Results are expressed as the mean + S.E. Statistically-significant difference at each time is shown as $a)(p<0.05)$, b) $(p<0.01)$ or $c)(p<0.001)$.

L-81 emulsion, was administered with the VEA preparation. As shown in Fig. 6 (A), VE was hardly observed in the thoracic lymph after the intraduodenal simultaneous administration of VEA/PC/MCTG preparation and L-81 emulsion, while the lymphatic transport was evident in the control experiment. On the other hand, no change in the plasma concentration of VE was observed after the administration of VEA/PC/MCTG preparation with or without L-81 emulsion in the lymph-fistula rat (Fig. 6 (B)), indicating that the transport of the vitamin by the portal route is not increased by inhibition of the lymphatic transport.

\section{Discussion}

VEA is a derivative of $\mathrm{VE}$ and is more stable against oxidation than VE. It has been reported that orally-administered VEA was hydrolyzed by the presence of bile ${ }^{1)}$ and pancreatic juice ${ }^{2,3)}$ in the intestinal lumen before the passage of intestinal mucosa and that the absorption mechanism of VE was similar to that of fat absorption. ${ }^{13)}$ Recently, in order to enhance the absorption of poorly water-soluble drugs, the use of lipoidal preparations has become of major interest. We also have shown that lecithindispersed aqueous preparation of VEA is an effective dosage form to enhance the absorption of VE and that lecithin is an important compo- nent for the enhancement. ${ }^{8)}$ In this study, we investigated the effect of modification of VEA preparations on the rate and the extent of systemic absorption and lymphatic transport of VE.

Endogenous VE concentration in the rat was $3-4 \mu \mathrm{g} / \mathrm{ml}$, while those in the dog and the human were $10-12 \mu \mathrm{g} / \mathrm{ml}^{14)}$ and $5-9 \mu \mathrm{g} / \mathrm{ml}$ (unpublished data), respectively, indicating large species differences. However, since the time course of the plasma concentration of endogenous VE was almost constant in the rat, the change in the plasma concentration of VE after the administration of VEA preparations can be estimated by subtraction of endogenous VE (0-time value in each rat) from the plasma concentration determined.

Figures 2 and 3 showed that the plasma concentration and $A U C$ of VE became highest in VEA/PC/MCTG (5/16/1) preparation, suggesting some action of MCTG. While the addition of MCTG to VEA/PC preparation changed the liposome structure to emulsions with relatively large particle size $(1-2 \mu \mathrm{m})$, this size could be reduced to about $80 \mathrm{~nm}$ in the bile salt solution, suggesting that the VEA preparation is dispersed well as smaller particles in the duodenum by the action of bile. This would be favorable for the absorption. Our preliminary experiments showed that the accumulation of the vitamin in intestinal tissue was increased by the presence of MCTG in the preparation, suggesting some action of 
MCTG on the mucosal uptake process. Since the particle size of the preparation seems to enlarge with increasing MCTG content even in the bile salt solution, the most suitable ratio might be observed as the result of these two opposite effects. The reduced accumulation by the addition of excess MCTG confirms with this assumption.

In order to clarify what caused the difference in the plasma concentration of VE with and without MCTG, VEA preparations were intraduodenally administered in the thoracic lymph-fistula rat. Figures 4 and 5 show that VE was transported into the thoracic lymph in VEA/PC/MCTG preparation more than in VEA/PC preparation and that $\mathrm{VE}$ concentration in the plasma did not increase even after the administration of VEA preparations in the lymph-fistula rat. Thus, it is suggested that the lymphatic route mainly contributed to the absorption of VE irrespective of whether or not MCTG was present and that the increase in the plasma concentration of VE by VEA/PC/MCTG preparation was due to the increase in the lymphatic transport of VE. GalloTorres et al. ${ }^{15)}$ reported that the intestinal absorption of VE was more enhanced by the solubilization in MCTG than in long-chain triglycerides. Some possible mechanism of absorption enhancement by MCTG and medium-chain fatty acids have been proposed. Gallo-Torres et al. ${ }^{15)}$ pointed out that medium-chain fatty acids are approximately 100 times more soluble in water than long-chain fatty acids, which would create suitable conditions for the faster uptake of VE. Hollander ${ }^{16)}$ showed that caprylic acid influenced the rate of lymphatic apprearance of vitamin A. Concerning the membrane premeability, medium-chain fatty acid was shown to affect the membrane fluidity. ${ }^{17)}$ However, the role of MCTG in the enhancement of VE absorption by VEA/PC/MCTG preparation remains unsolved at present.

The carrier for the lymphatic transport of water-insoluble compounds such as VE is mainly chylomicrons formed in the smallintestinal epithelium. ${ }^{18)} \mathrm{L}-81$, a lipophilic nonionic surfactant, has been shown to inhibit the synthesis and the transport of chylomicrons in the intestinal mucosa. ${ }^{10)}$ As shown in Fig. 6
(A), the transport of $\mathrm{VE}$ in the thoracic lymph was remarkably inhibited by the simultaneous administration of L-81. It is suggested that the chylomicron is essential to the absorption of $\mathrm{VE}$ from the VEA preparation containing MCTG.

In conclusion, VEA/PC/MCTG preparation is a very useful dosage form to enhance the $a b-$ sorption of VE, and MCTG as well as PC in the preparation is important. The increase in the plasma concentration of VE is due to the increase in the lymphatic absorption, where chylomicrons are essential.

\section{References}

1) M. T. MacMahon and G. R. Thompson: Lymphatic and portal venous transport of $\alpha$-tocopherol and cholesterol, Eur. J. Clin. Invest., 1, 288-294 (1971).

2) H. G. Gallo-Torres: Obligatory role of bile for the intestinal absorption of vitamin E, Lipids, 5, 379-384 (1970).

3) D. P. R. Muller, J. A. Manning, P. M. Mathias and J. T. Harries: Studies on the intestinal hydrolysis of tocopheryl esters, Int. J. Vit. Nutr. Res., 46, 207-210 (1976).

4) D. Hollander, E. Rim and K. S. Muralidahara: Mechanism and site of small intestinal absorption of $\alpha$ tocopherol in the rat, Gastroenterology, 68, 1492-1499 (1975).

5) M. Nagata, T. Yotsuyanagi, M. Nonomura and K. Ikeda: Coagulation recovery after warfarin-induced hypoprothrombinaemia by oral administration of liposomally-associated vitamin $\mathrm{K}_{1}$ to rabbits, J. Pharm. Pharmacol., 36, 527-533 (1984).

6) T. Higaki, I. Kishimoto, H. Komatsu, M. Hashida and H. Sezaki: Effect of medium-chain glycerides on the intestinal absorption and the hepatobiliary transport of phenol red, Int. J. Pharmaceut., 36, 131-139 (1987).

7) M. Sekine, K. Terashima, K. Nishimura, R. Okada and S. Awazu: Improvement of bioavailability of poorly absorbed drugs. II. Effect of medium chain glyceride base on the intestinal absorption of cefmetazol sodium in rats and dogs, J. Pharmacobio-Dyn., 8, 286-295 (1985).

8) T. Kimura, E. Fukui, A. Kageyu, H. Kurohara, Y. Kurosaki, T. Nakayama, Y. Morita, K. Shibusawa, S. Ohsawa and Y. Takeda: Enhancement of oral bioavailability of $d$ - $\alpha$-tocopherol acetate by lecithin-dispersed aqueous preparation containing medium-chain triglycerides in rats, Chem. Pharm. Bull., 37, 439-441 (1989).

9) J. L. Bollman, J. C. Cain and J. C. Grindlay: Techniques for the collection of lymph from the liver, small intestine, or thoracic duct of the rat, J. Lab. Clin. Med., 33, 1349-1352 (1948).

10) P. Tso and S. R. Gollamudi: Pluronic L-81: A potent inhibitor of the transport of intestinal chylomicrons, $\mathrm{Am}$. J. Physiol., 247, G32-G36 (1984). 
11) P. Tso, K. L. Buch, J. A. Balint and J. B. Rodgers: Maximal lymphatic triglyceride transport rate from the rat small intestine, Am. J. Physiol., 242, G408-G415 (1982).

12) P. Tso, J. A. Balint and J. B. Rodgers: Effect of hydrophobic surfactant (Pluronic L-81) on lymphatic lipid transport in the rat, Am. J. Physiol., 239, G348-G353 (1980).

13) Y.-F. Shiau: Mechanism of intestinal fat absorption, Am. J. Physiol., 240, G1-G9 (1981).

14) T. Tokumura, Y. Machida, Y. Tsushima, M. Kayano and T. Nagai: Intestinal absorption of tocopherol in beagle dog and effect of dosage form, Chem. Pharm. Bull., 35, 4592-4596 (1987).

15) H. E. Gallo-Torres, J. Ludorf and M. Brain: The effect of medium-chain triglycerides on the bioavailability of vitamin E, Int. J. Vit. Nutr. Res., 48, 240-249 (1978).

16) D. Hollander: Retinol lymphatic and portal transport: Influence of $\mathrm{pH}$, bile and fatty acids, Am. J. Physiol., 239, G210-G214 (1980).

17) N. Yata, W. M. Wu, R. Yamajo, T. Murakami, Y. Higashi and T. Higuchi: Enhanced rectal absorption of sodium ampicillin by $\mathrm{N}$-acyl derivatives of collagen peptide in rabbits and rats, J. Pharm. Sci., 74, 1058-1061 (1985).

18) I. R. Peake, H. G. Windmueller and J. G. Bieri: A comparison of the intestinal absorption, lymph and plasma transport and tissue uptake of $\alpha$ - and $\gamma$-tocopherols in the rat, Biochim. Biophys. Acta, 260, 679-688 (1972). 